\title{
Políticas educativas y actitudes relacionadas con la ciencia en estudiantes de bachillerato de Yucatán
}

\author{
Ángel Martín Aguilar Riveroll \\ aguilarr@correo.uady.mx \\ https://orcid.org/0000-0001-7247-2224 \\ Universidad Autónoma de Yucatán \\ Roger Jesús González González \\ rogr.gonzalez@gmail.com \\ https://orcid.org/0000-0003-2876-3539 \\ Universidad Pedagógica Nacional, Unidad 31-A \\ Galo Emanuel López Gamboa \\ galo.lopez@correo.uady.mx \\ https://orcid.org/0000-0001-5581-7489 \\ Universidad Autónoma de Yucatán
}

\section{RESUMEN}

Este estudio presenta los resultados de un análisis realizado para conocer la actitud hacia la ciencia de estudiantes yucatecos que cursan actualmente el bachillerato en diversos municipios del estado. Los resultados encontrados permiten tener un panorama más amplio de la actitud que tienen los jóvenes hacia la enseñanza de la ciencia, la imagen de la ciencia, la incidencia social de la ciencia y el conocimiento científico y técnico que se generan en Yucatán. Finalmente, con base en los resultados se puede concluir que los participantes tienen una actitud positiva hacia la ciencia y la consideran valiosa y de relevancia para el desarrollo del estado, por lo que es necesario el planteamiento de políticas educativas innovadoras encaminadas al fomento de vocaciones científicas en Yucatán.

Palabras clave: políticas educativas; actitud; ciencia; bachillerato; Yucatán. 


\title{
Educational policies and attitudes related to science in high school students in Yucatán
}

\begin{abstract}
ABSCTRACT
This study presents the results of an analysis carried out to know the attitude towards science of Yucatecan students who are currently studying high school in various municipalities of the state. The results found allow to have a broader panorama of the attitude that young people have towards the teaching of science, the image of science, the social impact of science and the scientific and technical knowledge that are generated in Yucatan. Finally, based on the results, it can be concluded that the participants have a positive attitude towards science and consider it valuable and relevant for the development of the state, which is why it is necessary to propose innovative educational policies aimed at promoting scientific vocations. in Yucatan.
\end{abstract}

Keywords: Educational policies, attitude, science, high school, Yucatán.

Artículo recibido: 30 noviembre. 2021 Aceptado para publicación: 29 diciembre 2021 Correspondencia: aguilarr@ correo.uady.mx Conflictos de Interés: Ninguna que declarar 


\section{INTRODUCCIÓN}

El desarrollo de la ciencia se encuentra ligado históricamente a los valores de la burguesía, y se vincula con los intereses de las clases dominantes (Ander-Egg, 2004). Esta afirmación es relevante pues en la actualidad la ciencia se relaciona al paradigma dominante, el neoliberalismo, la colonialidad y la modernidad, relegando del acceso al conocimiento científico a sectores históricamente vulnerados como son los indígenas, las mujeres y los jóvenes. En este sentido, estudios como el de Lara (2009) reflejan la necesidad de fortalecer la relación entre políticas públicas, educación, ciencia y tecnología pues "es indudable que el fomento a la ciencia y la tecnología se presenta como un elemento primordial para el desarrollo de las naciones, de ahí que las políticas públicas aplicadas en este tenor sean tan urgentes" (p. 17).

En los últimos años estudios como los de Schibeci, (1984), Shrigley y Koballa (1984), Acevedo (1993), Vázquez y Manassero, (1997), Leyton, et al. (2010) o Rodríguez, et al. (2007) se han orientado a definir instrumentos, metodologías y evaluaciones de las percepciones y actitudes de la población en general respecto a la ciencia, siendo escazas las investigaciones enfocadas en conocer la actitud de estudiantes de bachillerato respecto a los procesos de enseñanza, imagen, la influencia en la sociedad y las características generales de la ciencia, particularmente en el estado de Yucatán para el planteamiento de políticas educativas, científicas y tecnológicas que sean consistentes con estas actitudes. Es de destacar que en 2010, la Organización de Estados Iberoamericanos a través de los diferentes ministerios, secretarías e institutos que la conforman propuso impulsar el proyecto Metas educativas 2021, que entre sus principales objetivos busca "promover la alfabetización científica y estimular en los jóvenes la vocación por el estudio de las ciencias y la tecnología, a la vez que la independencia de juicio y un sentido de la responsabilidad crítica” (p.259), siendo una de las principales líneas de acción para conseguir dicho objetivo "realizar estudios acerca de la percepción social de la ciencia y de las capacidades de los países iberoamericanos en campos de ciencia básica, en disciplinas emergentes y en áreas de fuerte impacto" (p. 261). A partir de ello, se establece como una política pública internacional el desarrollo de estudios encaminados a conocer la percepción que tiene la sociedad iberoamericana acerca de la ciencia (Organización de Estados Iberoamericanos para la Educación, la Ciencia y la Cultura, 2010). 
Para el caso de México, la Ley de Ciencia y Tecnología que fue sancionada en el Diario Oficial de la Federación el 5 de junio de 2002, señala que el estado debe contar con una política que promueva la conformación de un Sistema Nacional de Ciencia y Tecnología para incrementar la capacidad científica, tecnológica que permita resolver diferentes problemas nacionales (Secretaría de Gobernación, 2015).

En ese sentido se espera que la masa crítica especializada aumente de forma gradual y se incorpore a programas de formación de alto nivel a través de posgrados de calidad a nivel, primeramente, de maestría, pero especialmente de doctorado. Una de las formas de promover esta formación desde etapas tempranas, es mediante los semilleros de investigación. De acuerdo con la Universidad de Cali (2019), un semillero de investigación es un espacio conformado por al menos tres estudiantes, que permite desarrollar habilidades propias de la actividad investigativa que siente las bases a largo plazo de candidatos idóneos para emprender proyectos de investigación. Algunos de sus

\section{fines son (Universidad de Cali, 2019, s.p.):}

- Posibilitar la formación investigativa de los estudiantes que les permita incursionar creativamente en el mundo del conocimiento.

- Facilitar a los estudiantes un espacio de formación colectiva en investigación con sus iguales y con el acompañamiento de un docente.

- Iniciar desde una etapa temprana el proceso de formación de quienes más adelante serán los jóvenes investigadores y potenciales docentes investigadores de las Facultades.

- Fomentar el desarrollo de aptitudes y actitudes científicas.

- Promover la capacidad de trabajo en equipo y del trabajo interdisciplinario.

- Promover la capacidad investigativa en el saber específico.

- Desarrollar actitudes y aptitudes para aprender a emprender proyectos de investigación.

- Desarrollar capacidades para participar activamente en los procesos de investigación adelantados en la Universidad.

Por lo anterior, los semilleros de investigadores resultan un insumo fundamental para la ciencia de un país y una estrategia de política publica para el fomento científico. 


\subsection{La ciencia en la educación media superior en México}

Los carentes resultados obtenidos en pruebas estandarizadas como es la prueba PISA (Programme for International Student Assessment), dirigida a estudiantes de 15 años de edad que se encuentran cursando el primer ciclo de la educación media superior o están por ingresar a ella, hacen pensar que la relación entre ciencia y la educación media superior en México no está teniendo los resultados esperados, pues en los últimos registros que se tienen de 2015, en el área de ciencias México ocupó el lugar 57 de 69 países participantes en el estudio (Instituto Nacional para la Evaluación de la Educación [INEE], 2016).

Son múltiples las variables que influyen en los resultados obtenidos en pruebas estandarizadas, así como en una percepción poco favorable de los jóvenes mexicanos hacia la ciencia y la tecnología, que Suárez (1996) sintetiza en tres factores fundamentales: 1) la diversidad de programas de estudio y tipos de bachillerato en el país es muy amplia, lo cual ocasiona que no existan lineamientos únicos ni se trabaje de forma conjunta en la promoción de la ciencia en el aula y el desarrollo de competencias científicas en los estudiantes; 2) el lugar la formación docente, pues en muchos casos las competencias docentes para la enseñanza de las ciencias son carentes, no existe una especialización del profesorado para fortalecer los conocimientos y habilidades que poseen para la enseñanza de temas científicos en el bachillerato, la formación básica del profesorado no permite a los profesionales de la educación ir más allá de los libros de textos y los planteamientos curriculares básicos y 3) el sistema educativo de nivel medio superior en México no se encuentra debidamente equipado para el desarrollo de actividades relacionadas con la ciencia, el trabajo en laboratorios o la interacción de los estudiantes con la tecnología y los sistemas digitales.

\subsection{Políticas educativas relacionadas con el desarrollo de actitudes científicas}

En el contexto de Yucatán se hace necesario el diseño e implementación de nuevas políticas públicas orientadas al fomento de la ciencia y la tecnología, ya que las acciones que se emprenden actualmente en materia de política educativa, científica y tecnológica, no son acordes a las necesidades del contexto y las características identitarias de la región (González González González, 2020; González González y Cisneros-Cohernour, 2020). Esta afirmación se puede constatar con algunos resultados de evaluaciones realizadas a los jóvenes yucatecos como la prueba PLANEA (Plan Nacional para la Evaluación de los 
Aprendizajes) 2015, administrada a 238 estudiantes de nivel medio superior en Yucatán. Se encontró que en el área de matemáticas el 51.4\% de los estudiantes se ubican en el nivel 1, el mínimo nivel esperado en estudiantes de este nivel educativo, seguido por el $30.2 \%$ en el nivel 2, $12.4 \%$ en el nivel 3 y únicamente el $6.1 \%$ en el nivel 4, el más elevado en esta escala que mide las competencias de los estudiantes en el área de matemáticas (Instituto Nacional para la Evaluación de la Educación, 2015).

Si bien en las últimas décadas México ha invertido en materia de Ciencia, Tecnología e Innovación, la velocidad con la que el presupuesto se ha destinado no ha sido suficientemente expedito en relación con otras naciones, pues la evidencia internacional señala que es conveniente contar con al menos el 1\% del Producto Interno Bruto (PIB) mientras que en nuestro país dicho indicador es el más bajo de las naciones integrantes de la Organización para la Cooperación y el Desarrollo Económicos (OCDE), e incluso menor al promedio latinoamericano (CONACYT, 2014 en SHCP, 2015). Entre las razones por las cuales el gasto en investigación científica y desarrollo experimental (GIDE) presenta ese nivel de atraso, reside en

el rezago en las capacidades de los actores nacionales que promueven el desarrollo científico, tecnológico y de innovación de México. Así entonces, se vuelve de interés general como problema público el fortalecer a los actores que dan vida al Sistema Nacional de Ciencia, Tecnología e Innovación (SNCTI) haciéndose necesarios programas presupuestarios como el F002, Apoyos para actividades científicas, tecnológicas y de innovación (SHCP, 2015, p. 4).

Algunos de los antecedentes en materia de fomento a las vocaciones científicas en nuestro país, se encuentran en el Programa de Apoyos para Actividades Científicas Tecnológicas y de Innovación del CONACYT. El cual tiene a su vez, como referente más remoto en la Ley de Ciencia y Tecnología de 2002, la cual indica en su artículo $2^{\circ}$, fracción I que se deberá "Incrementar la capacidad científica, tecnológica, de innovación y la formación de investigadores y tecnólogos para resolver problemas nacionales fundamentales, que contribuyan al desarrollo del país y a elevar el bienestar de la población en todos sus aspectos".

De acuerdo con datos de la Auditoría Superior de la Federación (2015), después de la publicación de la Ley de Ciencia y Tecnología, en 2008 se publicó el Programa Especial 
de Ciencia, Tecnología e Innovación (PECiTI) 2008-2012, en el que se incluyó el concepto de innovación con el propósito de integrar a los diferentes actores de ciencia y tecnología que pudiera coadyuvar para hacer más eficientes los procesos de producción, tomando como nombre Sistema Nacional de Ciencia, Tecnología e Innovación (SNCTI). En 2014, el PECiTI se convirtió en el referente de los objetivos y estrategias para la consolidación de las capacidades en ciencia, tecnología e innovación. Para llevar a cabo dicha tarea, el CONACYT implementó el programa presupuestario F002 “Apoyos institucionales para actividades científicas, tecnológicas y de innovación”, con el propósito de que los miembros del SNCTI generen, fortalezcan y articulen capacidades científicas y tecnológicas (ASF, 2015, p. 2).

Desde entonces, en los últimos 18 años se ha operado el Programa de Apoyos F002 con sus respectivas reglas de operación emitidas en el Diario Oficial de la Federación en los sexenios de Vicente Fox Quesada (2000-2006), Felipe Calderón (2006-2012), Enrique Peña Nieto (2012-2018) y recientemente, Andrés Manuel López Obrador (2018-2024).

En cuanto al concepto de actitud hacia la ciencia, que realmente refleja el interés más allá de los resultados de pruebas estandarizadas, Ander - Egg (2004) lo define como "la predisposición a detenerse frente a las cosas para tratar de desentrañarlas” (p. 121), compuesta por una gran parte de curiosidad de los individuos y el interés por conocer más acerca de los fenómenos que los rodean.

Por otro lado, Rodríguez, et al. (2007) proponen que el concepto de actitud se puede considerar como una causa del aprendizaje, pues afirman que una actitud positiva puede llegar a favorecer el aprendizaje de la ciencia y una actitud negativa puede llegar a dificultarlo.

La actitud frente a la ciencia es un concepto ampliamente abordado en el mundo y México, sin embargo, después de una extensa revisión de la literatura, no se encontraron estudios o diagnósticos enfocados en analizar la actitud que tienen hacia la ciencia los estudiantes de bachillerato en Yucatán y la propuesta de políticas educativas para su fortalecimiento y desarrollo. Debido a ello, se planteó como objetivo del presente estudio analizar las actitudes de estudiantes de bachillerato yucatecos, entre 14 y 18 años respecto a la ciencia, para el planteamiento de propuestas de políticas educativas que se correspondan con los resultados y las necesidades del contexto en Yucatán. 


\section{MATERIALES Y MÉTODO}

El presente es un estudio descriptivo en la modalidad de encuesta, ya que de acuerdo con Behar (2008) este tipo de estudios "sirven para analizar cómo es y cómo se manifiesta un fenómeno y sus componentes. Permiten detallar el fenómeno estudiado básicamente a través de la medición de uno o más de sus atributos" (p. 17). De forma adicional, el estudio se apoya en el análisis documental pues es una técnica que permite examinar información sin modificar la naturaleza del objeto de estudio, acercando la teoría a la investigación empírica (Vélez y Galeano, 2002).

\subsection{Participantes en el estudio}

En el estudio participaron de manera voluntaria 50 estudiantes de nivel medio superior, provenientes de diversos municipios del estado de Yucatán, los cuales se encuentran en primero, segundo o cursando el tercer año de la educación media superior en diversas modalidades de bachillerato y regiones del estado.

\subsection{Instrumento}

Para la recolección de los datos se utilizó el Protocolo de Actitudes Relacionadas con la Ciencia, adaptado por Rodríguez, et al. (2007). El instrumento se encuentra integrado por cuatro categorías principales, las cuales son: enseñanza de la ciencia, imagen de la ciencia, incidencia social de la ciencia y características de la ciencia.

Como mencionan los autores, la dimensión Enseñanza de la ciencia busca analizar las actitudes hacia la enseñanza y aprendizaje de la ciencia y la tecnología de los estudiantes. Por otro lado, la dimensión imagen de la ciencia se centra en investigar las actitudes que resultan de la interacción entre la sociedad, la ciencia y la tecnología. La tercera dimensión, se centra en explorar las actitudes relacionadas con temas específicos de ciencia y tecnología con incidencia social. Finalmente, con la cuarta categoría se pretende conocer la actitud hacia el conocimiento científico y técnico, abarcando actitudes hacia el trabajo de científicos y tecnólogos.

\section{RESULTADOS Y DISCUSIÓN}

El análisis de los resultados se realizó a través del Paquete Estadístico para las Ciencias Sociales (SPSS por sus siglas en inglés), se analizaron las variables sociodemográficas y los reactivos de acuerdo con las dimensiones propuestas en el Protocolo, los resultados se presentan a continuación. 
Como se observa en la Figura 1, en el estudio participaron 50 estudiantes de bachillerato cuyas edades oscilan entre los 14 y los 18 años. De igual forma es importante señalar que siete de los participantes cuentan con 14 años, siete jóvenes más con 16 años, cuatro de los participantes cuentan con 17 años y 31 estudiantes, la mayoría de los participantes, con 15 años. Es importante señalar que se registró la participación un joven de 18 años y que la media de edades de los participantes es de 15.2 años.

Figura 1. Distribución de participantes en el estudio por edad

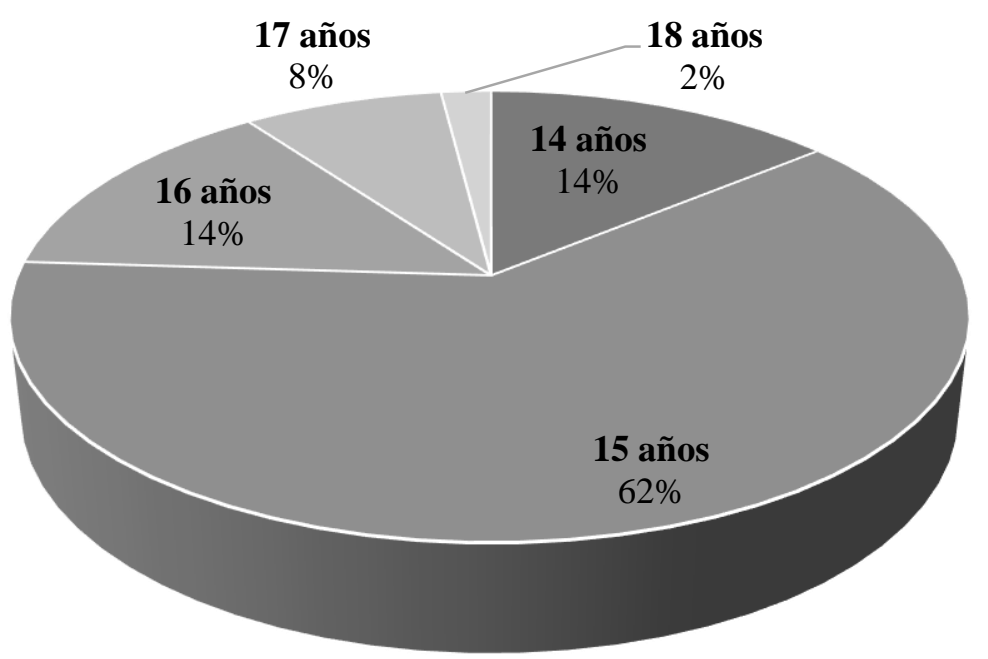

En cuanto al lugar de procedencia, en la Figura 2 se aprecia que el 54\% de los jóvenes reside en la ciudad de Mérida, mientras que el $46 \%$ de los participantes en el estudio son originarios de municipios del interior del estado como Valladolid, Tecóh, Maxcanú, Ticul, Halachó, Progreso y Hunucmá. Esta distribución es consistente con la distribución poblacional del estado de Yucatán, en la que el 50\% se encuentra en la capital (Mérida) y el resto de la población al interior del estado. Por otro lado, es importante mencionar que los municipios de los cuales provienen los participantes son representativos de todas las regiones de Yucatán, pues participaron jóvenes del centro, el sur, el oriente, el poniente y la costa del estado. 
Figura 2. Distribución de participantes en el estudio por municipio

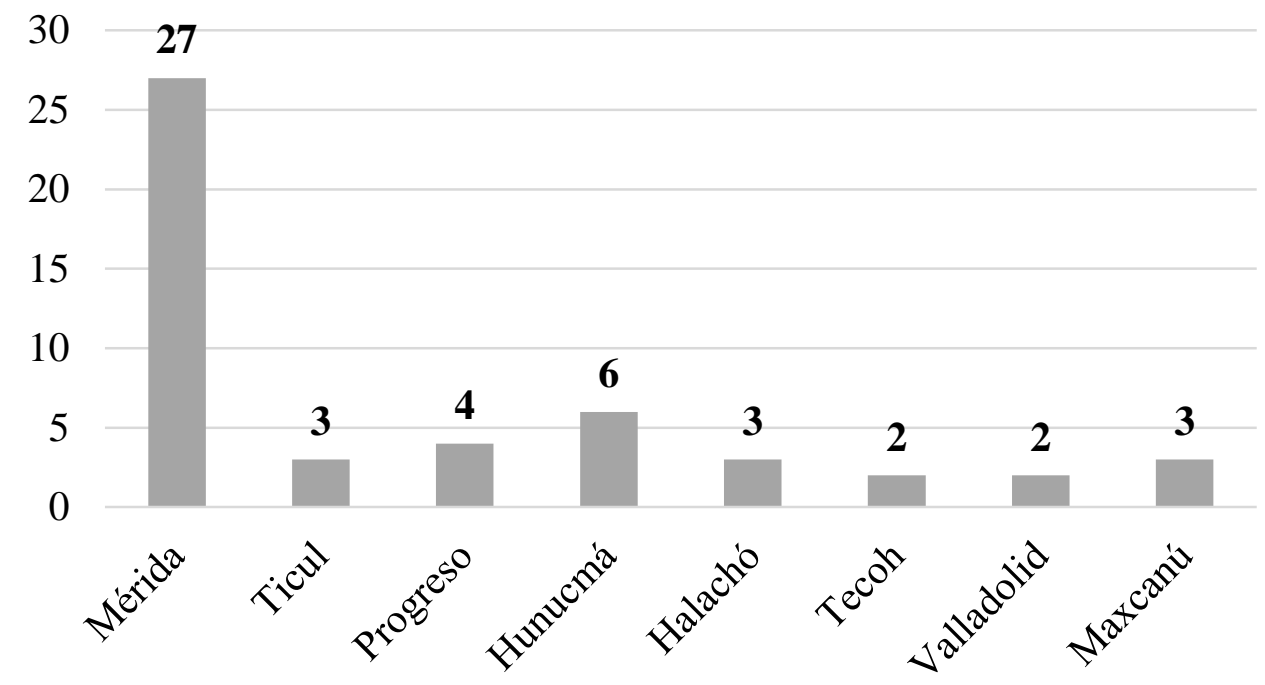

En el estudio participaron 37 estudiantes de primer año de bachillerato, 5 estudiantes de segundo año y 8 jóvenes de tercer año, estos datos se pueden apreciar de manera porcentual en la Figura 3. Esta diferencia en el porcentaje de participantes por grado escolar de la educación media superior obedece a múltiples factores, pero principalmente a los graves índices de abandono escolar que se atraviesan en México, pues estudios como el de Miranda López (2018) señalan que en el transcurrir de la educación media superior, más del $30 \%$ de los estudiantes matriculados desertan entre el primer y tercer año, principalmente por razones eminentemente económicas.

Figura 3. Distribución de participantes en el estudio por grado escolar

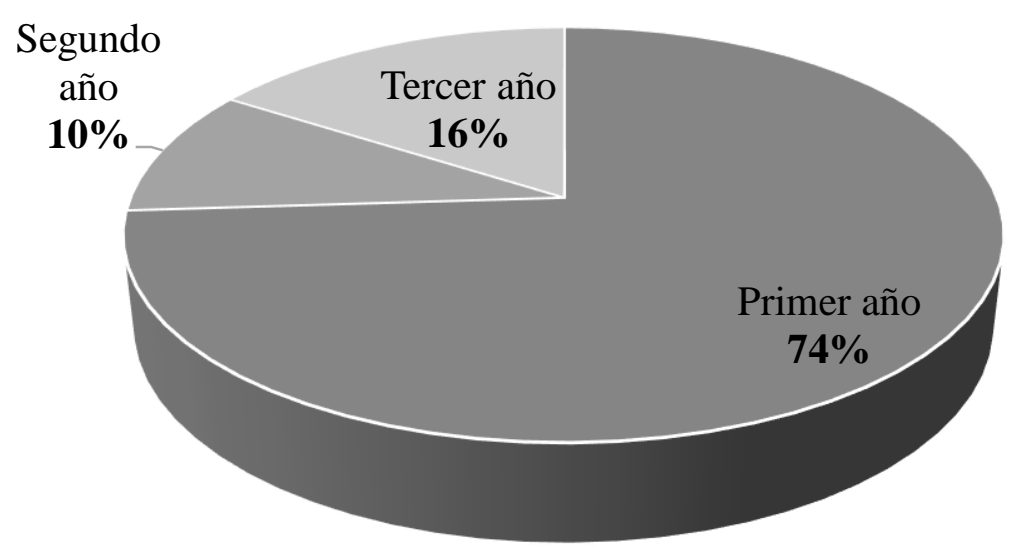




\subsection{Dimensión enseñanza de la ciencia}

Esta dimensión se enfocó en analizar la actitud de los estudiantes con relación a la enseñanza y aprendizaje de la ciencia en Yucatán. Se encontró en ítems como La ciencia es muy difícil de aprender, que el $46 \%$ de los participantes manifestaron estar en desacuerdo, mientras que el 30\% manifestó estar en total desacuerdo con la afirmación, con lo cual se puede afirmar que más del $70 \%$ de los participantes considera que la ciencia no es una disciplina difícil de aprender. Estos datos resultan interesantes, pues no son consistentes con otros estudios (Pozo y Gómez, 2006; Solbes, et al., 2007), en los que se reporta que para los jóvenes la ciencia es una disciplina difícil de aprender, sin embargo, se requieren investigaciones que profundicen en esta situación pues estos resultados se han encontrado sobre todo en contextos occidentales.

Con relación al reactivo Para destacarse en ciencia es necesario ser muy inteligente, el $58 \%$ de los participantes manifestó estar en desacuerdo, mientras que el $26 \%$ externó estar totalmente en desacuerdo con esta afirmación, ante lo cual se puede afirmar que el $84 \%$ de los estudiantes participantes considera que no es necesario ser muy inteligente para destacarse en la ciencia, es decir, los jóvenes tienen una actitud positiva con relación a las competencias y conocimientos que se requieren para destacar en el ámbito científico. Estos datos son consistentes con propuestas como la de Martín - Díaz (2004), quien afirma que la ciencia es parar todos, todos independientemente de sus capacidades, tienen derecho a comprender y tomar parte en las actividades científicas, además de que en México "la Constitución garantiza el derecho de toda persona a gozar de los beneficios del desarrollo de la ciencia y la innovación tecnológica” (Secretaría de Gobernación, 2021).

En el ítem Las asignaturas de ciencias son las peores el 18\% de los participantes manifestó estar en desacuerdo, mientras que el $82 \%$ de los jóvenes externó estar totalmente en desacuerdo con esta afirmación, es decir, el total de los participantes en el estudio tiene una actitud positiva respecto a las asignaturas de tipo científico que se imparten en la educación media superior en Yucatán.

Es importante considerar que este tipo percepciones y concretamente actitudes de los jóvenes yucatecos, reflejan la necesidad plantear políticas educativas en el marco del fomento de las vocaciones científicas, que se articulen con el currículo educativo y que 
desde los contenidos escolares propicien los conocimientos, habilidades y actitudes hacia la ciencia.

\subsection{Dimensión imagen de la ciencia}

Esta dimensión se configuró a través de ítems que exploraron la percepción de los jóvenes hacia la ciencia, a partir de la imagen que tienen de este constructo. En este sentido, se planteó el ítem La ciencia no le gusta a nadie, en el que el 30\% de los estudiantes afirmó estar en desacuerdo, mientras que el 64\% manifestó estar totalmente en desacuerdo con la afirmación, con lo que se puede afirmar que más del $90 \%$ de los participantes en el estudio se manifiestan en contra de esta aseveración.

En cuanto al reactivo La ciencia no es aburrida, se encontró que el $16 \%$ de los participantes están de acuerdo con este ítem, asimismo el 82\% de los jóvenes participantes externaron estar totalmente de acuerdo con la afirmación; prácticamente en su totalidad los participantes externaron que la ciencia no es aburrida, lo cual se vincula al ítem $L a$ ciencia es muy valiosa, pues se observó que en este reactivo el 100\% de los participantes manifestó una actitud positiva y valora la ciencia, lo cual es acorde a lo encontrado a través de instrumentos nacionales como la Encuesta Nacional sobre la Percepción Pública de la Ciencia y la Tecnología, desarrollada por el Consejo Nacional de Ciencia y Tecnología (CONACYT) en conjunto con el Instituto Nacional de Estadística, Geografía e Informática (INEGI), pues a través de este instrumento se puede afirmar que los mexicanos "han mostrado tener presente el beneficio económico y social que resulta de la actividad científica, lo cual puede ser un factor que contribuye a la percepción positiva que la población mexicana tiene sobre la actividad científica" (López Palau et al., 2018, p. 89).

\subsection{Dimensión incidencia social de la ciencia}

En esta categoría se analiza la relación de la ciencia y la tecnología con la sociedad, en ese sentido se puede afirmar que los participantes consideran que la ciencia es un factor determinante en el desarrollo y bienestar social, pues en reactivos como Gracias a la ciencia tenemos un mundo mejor se encontró que el $42 \%$ de los participantes está de acuerdo con la afirmación, que junto con el $58 \%$ que manifiestan estar totalmente de acuerdo, se puede comentar que el total de los participantes considera que la ciencia es un factor determinante para el bienestar social, representando un pilar para el desarrollo 
cultural, económico (Cañedo Andalia, 2001) y detonando la transformación en las estructuras sociales (Hualde Alfaro, 2012).

Por otro lado, en el ítem La gente vive más gracias a la ciencia, se encontró que el 54\% de los participantes está de acuerdo con esta afirmación, mientras que el $28 \%$ externó su total acuerdo, reflejando una perspectiva positiva acerca de los beneficios de la ciencia, lo cual es congruente con la necesidad percibida acerca del conocimiento científico, pues en el reactivo La ciencia es muy necesaria el $98 \%$ de los participantes externaron una actitud positiva (de acuerdo y totalmente de acuerdo) ante esta afirmación.

\subsection{Dimensión características de la ciencia}

Finalmente, esta categoría se integró por reactivos como La curiosidad es lo primordial de la ciencia, en donde el $44 \%$ de los participantes manifestó estar de acuerdo, adicionalmente el $26 \%$ manifestaron estar totalmente de acuerdo con el hecho de que la curiosidad es un factor determinante en el pensamiento científico.

Los últimos ítems analizados fueron La ciencia disminuye la curiosidad y En la ciencia es importante tener en cuenta las ideas nuevas, que se relacionan con el tema de la creatividad en el sector científico. En estos planteamientos se encontró que $96 \%$ de los participantes externó estar en desacuerdo y total desacuerdo con la primera afirmación de este párrafo, mientras que el $98 \%$ confirmó estar de acuerdo y totalmente de acuerdo con el hecho de que en la ciencia es importante tener presente las ideas nuevas, con lo que se pone de manifiesto la importancia del fomento a la creatividad para el éxito en la ciencia desde la perspectiva de los participantes.

\section{CONCLUSIONES}

Con base en el análisis realizado se puede afirmar que, los participantes en el estudio tienen en general una actitud positiva hacia el ámbito científico, la manera en la que se enseña, su relación con la sociedad y los atributos de esta.

Los participantes consideran que la ciencia es un agente de cambio social y que es necesaria para el desarrollo, por lo que no es imprescindible tener altas capacidades para desenvolverse en el ámbito científico, basta con interés y curiosidad por los fenómenos que rodean al ser humano para estudiarlos y comprenderlos. Esto se puede constatar con los resultados obtenidos en la dimensión, enseñanza de la ciencia, a través de la cual se pudo constatar que para destacarse en ciencia no es necesario ser muy inteligente. 
Yucatán se está convirtiendo en un polo científico en el país, muestra de ello son las diferentes políticas y la implementación de acciones como son la creación de Centros públicos de investigación, Instituciones públicas promotoras de la ciencia y la tecnología, así como el incremento de empresas de base tecnológica. Ante este escenario, sea hace necesario considerar que los estudiantes que en escasos quince años serán los encargados de generar ciencia y tecnología en la región, tienen una actitud positiva y una alta valoración de la ciencia, por lo que es imprescindible el planteamiento de políticas públicas y específicamente en materia educativa que prioricen el fomento de vocaciones científicas desde el currículo educativo a edades tempranas.

Finalmente, este estudio representa un primer acercamiento desde el contexto de Yucatán a la relación entre política educativa, actitud científica y fomento de vocaciones científicas, por lo que con base en los resultados se propone el desarrollo de un estudio posterior más profundo que permita captar las realidades que se viven en todo el contexto de Yucatán con relación fomento de la ciencia y la tecnología en jóvenes.

\section{LISTADO DE REFERENCIAS BIBLIOGRÁFICAS}

Acevedo, J.A. (2004). ¿Qué piensan los estudiantes sobre la ciencia?, un enfoque CTS. IV Congreso Internacional sobre investigación en la didáctica de las ciencias y las matemáticas. https://raco.cat/index.php/Ensenanza/article/view/21083

Ander - Egg, E. (2004). Técnicas de investigación social. Argentina: Colección política, servicios y trabajo social.

Auditoría Superior de la Federación (2015) Auditoría de Desempeño: 14-1-3890X-07042 al Programa de apoyos para actividades científicas, tecnológicas y de innovación

Autor. Recuperado de

https://www.asf.gob.mx/Trans/Informes/IR2014i/Documentos/Auditorias/2014_0429_a .pdf

Behar, D. (2008). Metodología de la investigación. España: Editorial Shalom.

Cañedo Andalia, R. (2001). Ciencia y tecnología en la sociedad: Perspectiva histórico $\begin{array}{llll}\text { conceptual. } & \text { ACIMED, }\end{array}$ http://scielo.sld.cu/scielo.php?script=sci_arttext\&pid=S1024$94352001000100005 \& \operatorname{lng}=\mathrm{es} \& \operatorname{lng}=\mathrm{es}$. 
Secretaría de Hacienda y Crédito Público (2015) Diagnóstico del Programa Presupuestario F002 “Apoyos para actividades científicas, tecnológicas y de innovación”. México: Autor.

González González, R. J. (2020). Justicia social e inequidad educativa en la formación científica y tecnológica (Tesis de Doctorado). Universidad Autónoma de Yucatán.

González González, R. J. y Cisnernos-Cohernour, E. J. (2020). Justicia Social e Inequidad en la Formación Científica y Tecnológica de Jóvenes Rurales en la Región Maya de

México: El Caso de Mex. Revista Internacional de Educación para la Justicia Social, 9(1), 19-39. https://doi.org/10.15366/riejs2020.9.1.001.

Hualde Alfaro, A. (2012). Reflexiones sobre la relación entre ciencias sociales y actores regionales en México. Frontera norte, 24(48), 93-119. http://www.scielo.org.mx/scielo.php?script=sci_arttext\&pid=S0187 $73722012000200004 \& \operatorname{lng}=$ es\&tlng=es.

Instituto Nacional para la Evaluación de la Educación (2015). Plan Nacional para la Evaluación de los Aprendizajes en la Educación Media Superior. México.

Instituto Nacional para la Evaluación de la Educación (2016). México en PISA 2015. 1a edición. México: Autor.

Lara, L. M. (2009). Acerca de la potencialidad secuestrada de la ciencia y tecnología. Por una idea del desarrollo multidimensional. En G. Sánchez, S. A. Figueroa, A. Vidales

(Eds.), La ciencia y tecnología en el desarrollo: Una visión desde América Latina México: Consejo Latinoamericano de Ciencias Sociales.

Leyton, D., Sánchez, C. y Ugalde, P. (2010). Estudio Percepción de los Jóvenes sobre la Ciencia y Profesiones científicas. Comisión Nacional de Investigación Científica y Tecnológica.

López Palau, N., Flores Gutiérrez, C. y Femat Flores, A. (2018). Opinión pública sobre el

investigador científico y la ciencia en México. Revista Ciencia, 69(1). 84 - 89. https://www.revistaciencia.amc.edu.mx/images/revista/69_1/PDF/OpinionPublic a.pdf 
Martín-Díaz, M. (2004). El papel de las ciencias de la naturaleza en la educación a debate. Revista Iberoamericana de Educación. http://www.rieoei.org/deloslectores/692MartinDiaz.PDF

Miranda López, F. (2018). Abandono escolar en educación media superior: conocimiento y aportaciones de política pública. Sinéctica Revista Electrónica de Educación, 51, 1 - 22. https://doi.org/10.31391/S2007-7033(2018)0051-010

Organización de Estados Iberoamericanos para la Educación, la Ciencia y la Cultura (2010). 2021 metas educativas, la educación que queremos para la generación de los bicentenarios. España: Autor.

Pozo, J. I. y Gómez, M. A. (2006). Aprender y enseñar ciencia. Del conocimiento cotidiano al conocimiento científico. España: Morata.

Rodríguez, W., Jiménez, R. y Caicedo-Maya, C. (2007). Protocolo de actitudes relacionadas con la ciencia: adaptación para Colombia. Psychologia. Avances de la disciplina, $1(2), 85-100$. https://www.redalyc.org/articulo.oa?id=297224996001.

Schibeci, R.A. (1984). Attitudes to science: an update. Studies in Science Education, 11(s.v.), 26 -59. https://doi.org/10.1080/03057268408559913.

Secretaría de Gobernación (2015). Ley de Ciencia y Tecnología. México: Diario Oficial de la Federación. http://www.ordenjuridico.gob.mx/Documentos/Federal/html/wo83111.html.

Secretaría de Gobernación (2021). Acuerdo por el que se reforma el Reglamento del Sistema Nacional de Investigadores. México: Diario Oficial de la Federación. https://www.dof.gob.mx/nota_detalle.php?codigo=5625348\&fecha=30/07/2021.

Shrigley, R. L., \& Koballa, T. R. (1984). Attitude measurement: Judging the emotional intensity of Likert-type science attitude statements. Journal of Research in Science Teaching, 21(2), 111-118. https://doi.org/10.1002/tea.3660210203

Solbes, J., Motserrat, R. y Furió, C. (2007). El desinterés del alumnado hacia el aprendizaje de la ciencia: implicaciones en su enseñanza. Didáctica de las ciencias experimentales y sociales, 21(s.v.), $91 \quad-\quad 117$. https://roderic.uv.es/handle/10550/20927. 
Suárez, L. (1996). Enseñanza de la Metodología de la ciencia en el Bachillerato. Perfiles Educativos, $\quad 18 \quad$ (73). Recuperado de https://www.redalyc.org/pdf/132/13207304.pdf.

Universidad de Cali (2019) ¿Qué es un Semillero de Investigación?. Colombia: Autor.

Recuperado de https://www.usbcali.edu.co/node/208

Vázquez, A. y Manassero, M. A. (1997). Una evaluación de las actitudes relacionadas con la ciencia. Enseñanza de las ciencias: revista de investigación y experiencias $\begin{array}{lllll}\text { didácticas, } & 15(2), & & 199 & \end{array}$ https://raco.cat/index.php/Ensenanza/article/view/21491.

Vélez, O.L. y Galeano, M.E. (2002) Estado del arte sobre fuentes documentales en investigación cualitativa, Medellín: Centro de Investigaciones Sociales y Humanas, Universidad de Antioquia. 